REVISTA DE DERECHO UNED, NÚM. 23, 2018

\title{
LA GESTIÓN SANITARIA EN ESPAÑA: NECESIDAD DE UN NUEVO MODELO DE FINANCIACIÓN Y PROVISIÓN DE LAS PRESTACIONES SANITARIAS*
}

HEALTH MANAGEMENT IN SPAIN: NEED FOR A NEW MODEL OF FINANCING AND PROVISION OF HEALTH BENEFITS

\section{José JEREZ IgLESIAS}

Economista, Graduado en Derecho, Master en Derechos Humanos

Doctorando Facultad de Derecho

Universidad Nacional de Educación a Distancia (UNED)

Resumen: La cuestión de la gestión sanitaria en España plantea interrogantes sobre cómo abordar las causas que inciden en sus síntomas de falta de sostenibilidad y como resolver sus ineficiencias más significativas, es decir, cómo encontrar modelos más eficientes de gestión de las prestaciones sanitarias para hacer sostenible el derecho a la prestación sanitaria de los ciudadanos.

Se propone una reforma del Sistema Nacional de Salud (SNS) con un modelo alternativo de financiación y provisión de las prestaciones sanitarias, basado en los principios de competencia, eficacia, eficiencia y libre elección de los asegurados entre centros concertados públicos y privados.

Palabras clave: Provisión sanitaria. Centros Concertados. Eficiencia. Libre elección. Copago. Ticket moderador.

* Este trabajo se realiza dentro del proyecto DER 2013-41462-R del Ministerio de Economía y Competitividad. 
Abstract: The issue of health management in Spain raises questions about how to address the causes that affect its symptoms of lack of sustainability and how to solve its most significant inefficiencies, that is, how to find more efficient models of health benefits management to make sustainable the right to health care for citizens.

A reform of the National Health System is proposed with an alternative model of financing and provision of health benefits, based on the principles of competence, effectiveness, efficiency and free choice of insured persons between public and private contracted centers.

Keywords: Health provision. Contracted centers. Efficiency. Free choice. Copay. Moderator ticket.

Recepción original: 07/02/2018

Aceptación original: 09/09/2018

Sumario: I. Introducción.-II. Situación actual del Sistema Nacional de Salud (SNS). II.1. Listas de espera excesivas. II.2. Desigual atención a los asegurados fuera de su autonomía de residencia. II.3. Dificultades de compensación económica entre sistemas autonómicos. II.4. Obsolescencia tecnológica sanitaria. II.5. Distorsión de la financiación autonómica.-III. Propuesta de un modelo alternativo de financiación y provisión de las prestaciones sanitarias. III.1. Principios en que se fundamenta la propuesta. III.2. Diagrama y características básicas del modelo. III.3. Centros concertados públicos y privados. III.4. La financiación. III.5. Una propuesta de copago. III.6. Ventajas del modelo propuesto.-IV. Conclusiones.V. Bibliografía.

\section{INTRODUCCIÓN}

El principal objetivo de esta colaboración es reflexionar sobre la necesidad de encontrar modelos de gestión de la financiación y provisión de las prestaciones sanitarias más eficientes para hacer sostenible el derecho a la prestación sanitaria de los ciudadanos, compatibilizando la reforma necesaria con el cumplimiento de los mandatos constitucionales del artículo $14 \mathrm{CE}$, que garantiza la igualdad de todos los españoles, y los del artículo 31.2. CE, que exige asignar equitativamente los recursos públicos con criterios de eficiencia y economía. 
Con esta finalidad investigamos la situación actual del Sistema Nacional de Salud (SNS) en España, y tratamos de objetivar lo que denominamos ineficiencias del sistema e identificamos las razones fundamentales para plantear una reforma profunda del sistema de financiación y provisión actual.

Formulamos nuestra propuesta de reforma del SNS, con un modelo alternativo de financiación y provisión de las prestaciones sanitarias, basado en los principios de competencia, eficacia, eficiencia y libre elección de los asegurados entre centros concertados públicos y privados. Se propone también la implantación de un sistema de copago farmacéutico distinto del actual y un nuevo sistema de tickets limitados para asistencia en atención primaria, especializada, urgencias, pruebas diagnósticas y terapéuticas, con un pequeño copago una vez agotada la dotación inicial de crédito de tickets sin pago.

\section{SITUACIÓN ACTUAL DEL SISTEMA NACIONAL DE SALUD}

El Sistema Nacional de Salud (SNS) es el resultado de la descentralización de las competencias sanitarias que se han ido modificando desde su origen en 1942 con el Seguro Obligatorio de Enfermedad, con la Ley de Bases de la Seguridad Social de 1963 y posteriormente con la Constitución española de 1978 (artículos 43, 49 y 53.3 CE), completado posteriormente con la Ley 14/1986, de 5 de abril, General de Sanidad, en la que se realiza una integración de todos los recursos públicos en el Servicio de Salud.

El Sistema Nacional de Salud, conjunto de Servicios de Salud de la Administración del Estado y de las Comunidades Autónomas, forman un órgano que pretende ser un conjunto coherente, armónico y solidario. Los principios generales son: Universalidad, Equidad social e interterritorial, Integración de todos los medios asistenciales, y Orientación al ciudadano: Derechos y deberes. Sus características más importantes son: Cobertura universal, Prestaciones amplias, Financiación pública, y Gestión descentralizada. Todas las Comunidades Autónomas recibieron las competencias en Sanidad e Higiene desde 1979 en adelante. La evolución del sistema sanitario público, desde un modelo basado en Seguros Sociales y centralizado hacia un Sistema Nacional de Salud descentralizado, ha tenido asociado diferentes modelos de financiación, determinados por los procesos de reforma hacía su actual configuración. Podemos objetivar dos procesos fundamentales: i) Universalización. La universalización

(C) UNED. Revista de Derecho UNED, núm. 23, 2018 
de la asistencia sanitaria, que determina la naturaleza de los recursos que financian la sanidad y ha propiciado la sustitución de cotizaciones sociales por impuestos generales. ii) Descentralización. La descentralización ha propiciado la integración de la financiación finalista de la asistencia sanitaria del Sistema Nacional de Salud en el sistema general de financiación de las Comunidades Autónomas. Desde el punto de vista de los modelos de financiación territorial de la sanidad pública española se pueden distinguir dos etapas bien diferenciadas. La primera abarca veinte años (1981-2001), la segunda etapa comienza con la publicación de la Ley 21/2001. La primera etapa estaba basada en la territorialización del gasto, mediante la descentralización de la gestión del gasto, que no llevó aparejada la descentralización de los ingresos y se financiaba la sanidad con un modelo específico mediante una transferencia finalista de la Administración General del Estado, utilizando distintos criterios de reparto, principalmente el de población. En la segunda etapa se integra la sanidad en el modelo general, al mismo tiempo que se crea un «espacio fiscal» que supone un cierto grado de descentralización, incorporando así la corresponsabilidad fiscal.

Es evidente que la crisis económica vivida en el mundo occidental entre 2008 y 2012 ha tenido unos efectos devastadores en la actividad económica, produciendo unas fuertes bajadas de inversiones, desencadenado un efecto dominó con cierres de empresas, desempleos masivos, bajadas de la demanda de bienes y servicios, y disminución de los ingresos fiscales. «Durante los primeros años de la crisis se fue profundizando en los principios generales del sistema de Salud: sostenibilidad, eficiencia e igualdad, teniendo en cuenta especialmente España, la responsabilidad de los profesionales en el control del gasto público referido a la salud...» ${ }^{1}$

Un análisis de su situación actual nos permite objetivar las ineficiencias del SNS más significativas:

\section{II.1. Listas de espera excesivas}

La situación de la lista de espera quirúrgica y de acceso a una consulta con un médico especialista en el Sistema Nacional de Salud a fecha 31 de diciembre de 2016, tiene una progresiva evolución al retraso y por tanto al crecimiento del número de personas en espera. Como pacientes pendientes de una intervención se entiende

1 MARCOS DEL CANO, A.M. y MÉNDEZ MARTÍN, J., «El Derecho a la Salud. Perspectivas tras la crisis». Revista de Derecho UNED, n. ${ }^{\circ}$ 19, 2016, pág. 166. 
aquellos que se les ha prescrito una intervención no urgente para la cual se tiene previsto el uso del quirófano y, además, están incluidos en un registro de pacientes pendientes de una intervención y la fecha de entrada en dicho registro es la de prescripción. Para los pacientes pendientes de una primera consulta en Atención Especializada, se utiliza el mismo criterio que para la lista de espera quirúrgica. Según la información facilitada por el Ministerio de Sanidad, la situación a 31 de diciembre de 2016 por Comunidades Autónomas el tiempo medio de espera en España para ser operado en el SNS es de 115 días (en junio de 2016 era de 83 días) y afectaba a 614.101 personas (en junio de 2016 eran 569.097 pacientes). ${ }^{2}$ La situación en el tiempo de espera para consultas externas y especialidades también ha empeorado hasta alcanzar los 72 días de media (en junio de 2016 eran 52 días). El número de pacientes pendientes de un especialista era de 45,66 por cada 1.000 habitantes. Las Comunidades que tienen mayor tiempo de espera para acceder a una consulta de especialista son: Cataluña, con 138 días, Canarias (117 días) y Aragón (79 días). Las que menos son: Melilla (21 días), País Vasco (27 días) y La Rioja (32 días). La variación de la lista de espera quirúrgica en las principales especialidades, en el periodo $2015-2016$ es la siguiente: Todas las especialidades: $+29 \%$, General y digestivo: $+38 \%$, Traumatología: $+28 \%$, Oftalmología: $+12 \%$.

Las listas de espera se ven influenciadas por el envejecimiento de la población, "aunque la longevidad no es un problema estrictamente, ya que mejora la esperanza de vida... (...), pero conlleva un mayor gasto sanitario y asistencial». ${ }^{3}$

\section{II.2. Desigual atención a los asegurados fuera de su autonomía de residencia}

Uno de los efectos más perversos del Sistema Nacional de Salud, es haberse convertido en diecisiete sistemas de salud, independientes entre sí, jerárquica y financieramente, lo que supone romper el principio constitucional de igualdad de los españoles ante la ley, «los españoles son iguales ante la ley, sin que pueda prevalecer discriminación alguna por razón de nacimiento, raza, sexo, religión, opinión o cualquier otra condición o circunstancia personal o social» (artículo $14 \mathrm{CE})$.

${ }^{2}$ Sistema de Información sobre listas de espera en el Sistema Nacional de Salud-SISLE-SNS, 2016.

3 MARCOS DEL CANO, A.M. y MÉNDEZ MARTÍN, J., «El Derecho a la Salud. Perspectivas tras la Crisis». Revista de Derecho UNED n. ${ }^{19}$, 2016, pág. 179.

(C) UNED. Revista de Derecho UNED, núm. 23, 2018 
Esta desigualdad se pone de manifiesto cuando un español residente en una comunidad autónoma, necesita ser atendido en un centro sanitario de otra comunidad, donde será atendido como un asegurado de otra comunidad, sin derecho a las mismas prestaciones de los asegurados residentes en esa comunidad. Y ello es debido a la autonomía financiera y de gestión en cada comunidad de las prestaciones del derecho de protección de la salud, por lo que económicamente atender a un usuario de otra comunidad supone un gasto que debería soportar la comunidad de origen del usuario, razón por la que la asistencia sanitaria se restringe a la mínima intervención, teniendo que recuperar el gasto por medio del sistema de compensación entre comunidades, cuyo funcionamiento tampoco es bueno como veremos.

\section{II.3. Dificultades de compensación económica entre sistemas autonómicos}

Con carácter extrapresupuestario y al objeto de garantizar la cohesión y equidad en el Sistema Nacional de Salud, mediante la cobertura de los desplazamientos entre comunidades autónomas de personas que gozan de la condición de asegurado, se creó el Fondo de Garantía Asistencial. El Fondo está destinado a la compensación entre las comunidades autónomas por las actuaciones que sus servicios asistenciales y de la suplementaria a las personas que gozan de la condición de asegurada en el Sistema Nacional de Salud en sus desplazamientos temporales. A tal fin se creó una tarjeta sanitaria individual del Sistema Nacional de Salud, con el fin de realizar una adecuada gestión y seguimiento del Fondo de Garantía Asistencial. A fecha de hoy, la realidad es que el Fondo de Cohesión Asistencial, poco a poco, se ha ido quedando sin financiación del Estado (93,3 millones en 2012, 2,3 millones en 2015, 0 millones en 2016), pasando a ser de exclusiva «naturaleza extrapresupuestaria» desde 2016. Las comunidades comunicarán el importe que les ha supuesto atender a pacientes de otras regiones, se nivelarán los saldos positivos y negativos y el dinero se ingresará en una cuenta de la Secretaría General del Tesoro que gestionará el Ministerio de Sanidad. En teoría, si las autonomías no dispusieran los importes, el gobierno podría deducir o retener cantidades de su financiación. «Hace al menos tres años que el Ministerio de Sanidad no actualiza el fondo de cohesión sanitaria, una partida que tiene como fin compensar la asistencia sanitaria a pacientes que se deriven de una comunidad autónoma 
distinta de aquella donde viven, en muchas ocasiones por casos de enfermedades raras. ${ }^{4}$

\section{II.4. Obsolescencia tecnológica sanitaria}

España no cumple con las directrices europeas del COCIR (Comité Europeo de Coordinación de la Industria de Electro-medicina, Radiología y Cuidado de Salud). En equipos de alta tecnología, según SERAM (Sociedad Española de Radiología Médica), solo un $34 \%$ de las tomografías computarizadas (TC) tenías menos de cinco años de antigüedad, el 44\% tenía entre cinco y diez años y el $23 \%$ incluso hasta más de diez años. Las resonancias magnéticas, un 33\% tiene más de 10 años y un $25 \%$ tiene menos de cinco años. "Si en el caso de las TC España ocupa el quinto lugar por la cola en Europa en obsolescencia tecnológica con las RM aparece en el último lugar. La solución y el problema es cómo financiar el plan: ¿Cómo pagarán los hospitales? La situación no es crítica, pero el problema es muy serio». ${ }^{5}$

Para el presidente de la Sociedad Española de Electro-medicina e Ingeniería Clínica (SEEIC), Lucinio Manzanares «nunca hay planes al respecto a medio-largo plazo, ni capital finalista para llevarlos a cabo. Además, las realidades autonómicas son muy distintas. La explicación reside en el modelo que España utiliza para gestionar la renovación de su parque tecnológico sanitario. Es lamentable que aún tengamos que alegrarnos cuando, por ejemplo, una provincia española adquiere su primer PET-TC, una tecnología que debería estar en el día a día de todo paciente oncológico. No en cada hospital, pero si en el entorno cercano al paciente». ${ }^{6}$

\section{II.5. Distorsión de la financiación autonómica}

El modelo de financiación actual de las Comunidades Autónomas, es motivo de insatisfacción para todos. Nadie está conforme con la fórmula de asignación de recursos financieros desde el Estado a las autonomías. Posiblemente uno de los capítulos que genera más conflictividad es el correspondiente a la financiación de las prestaciones sanitarias del derecho a la protección de la salud de los ciu-

${ }^{4}$ redaccionmedica.com, página consultada 28-12-17.

5 GAYETE, A., Presidente SERAM, Diario Médico 11-06-17.

${ }^{6}$ MANZANARES, L., Diario Médico, 11-06-17. 
dadanos. Las distorsiones entre autonomías se producen por una diversidad de causas de distinta naturaleza.

Así, la dispersión del territorio y la densidad de población por kilómetro cuadrado, que provocan desplazamientos largos, obligación de mantener consultorios o urgencias con poca actividad, las políticas de inversiones en nuevos centros y equipos tecnológicos sanitarios, que agotan los presupuestos o retrasan «sine die» esas necesarias inversiones. Las políticas retributivas del personal sanitario de cada comunidad que genera agravios comparativos, etc.

El sistema de financiación no satisface a nadie. Es muy probable que sacando la financiación de la sanidad de los presupuestos autonómicos, para ser financiada la prestación de los servicios sanitarios directamente desde la Administración del Estado, se solucionaría en gran parte el problema de la asignación financiera sanitaria a las Comunidades Autónomas.

Además, de las ineficiencias descritas, en nuestra opinión, existen razones de necesario cumplimiento que justificarían la necesidad de una reforma:

a) Necesidad de financiar el mismo coste por unidad de prestación, tanto en la prestación de la sanidad pública como en la privada

La financiación del Derecho de Protección de la Salud reconocido en la Ley 14/1986, de 25 de abril, General de Sanidad, con fundamento en el principio informador constitucional que declara el artículo 43 de la Constitución española (CE) constituye un deber para la Administración del Estado. Del mismo modo constituye un deber también para la Administración del Estado cumplir lo dispuesto en el artículo 31.2 CE que dice: «El gasto público realizará una asignación equitativa de los recursos públicos, y su programación y ejecución responderán a los criterios de eficiencia y economía.» ${ }^{7}$

Este artículo 31.2 CE está incluido en la Sección 2. ${ }^{a}$ del Capítulo Segundo del Título I, y por tanto, le es de aplicación también lo dispuesto en el artículo 53.1 CE que dice: «Los derechos y libertades reconocidos en el Capítulo segundo del presente título vinculan a todos los poderes públicos. Solo por Ley que en todo caso deberá respetar su contenido esencial, podrá regularse el ejercicio de tales

${ }^{7}$ Artículo 31.2 CE. 
derechos y libertades, que se tutelaran de acuerdo con lo previsto en el artículo 161.1.a).»8

Por lo expuesto, el Estado tiene el deber de financiar la producción de las prestaciones sanitarias teniendo en cuenta los criterios de eficiencia y economía del gasto público que le impone la Constitución española. Aplicar estos criterios de eficiencia y economía implica financiar el coste de producción de las prestaciones sanitarias, al mismo o parecido coste que se obtendría en un modelo de provisión competitiva con operadores públicos y privados, donde el asegurado pudiera elegir libremente el centro, público o privado, en el que obtuviera la oferta más eficaz en cantidad y calidad de los servicios sanitarios, en el menor tiempo posible, y por tanto sin esperas y dilaciones no admisibles. En la situación actual, el deber del Estado se convierte también en necesidad para hacer sostenible el Sistema Nacional de Salud, y ello requiere buscar soluciones y pactos políticos para llevarlas a efecto.

«Si el modelo de dirección pública de la gestión sanitaria ha sido incapaz de controlar el crecimiento del gasto, cabría preguntarnos si no ha llegado el momento de, conservando la provisión pública que nadie discute, introducir criterios de mercado en la producción de la sanidad, que es un bien privado, lo que podría dar lugar a eliminar las ineficiencias que se producen cuando la producción de un bien privado se traspasa a la esfera del sector público.» ${ }^{9}$

«En un contexto de no mercado, las internalidades aumentan de modo excesivo los costes del organismo, desplazando hacia arriba sus curvas de ofertas, por encima de lo que sería técnicamente factible, dando lugar a unos costes unitarios más altos y a unos niveles más reducidos de producción real del no mercado que los que serían socialmente eficientes, existiendo, por tanto, un fallo del no mercado», por ejemplo, «el crecimiento presupuestario (más es mejor) y el progreso tecnológico (lo nuevo y lo complejo es mejor).» ${ }^{10}$

«En los servicios de asistencia sanitaria suministrados por los países occidentales, la fracción de los costes médicos que asume el asegurado es tan pequeña (o nula) que prácticamente no tiene incentivos para limitar los costes. Así, las fuerzas del mercado que garantizan habitualmente la eficiencia económica, simplemente no entran en juego.»11

8 Artículo 53.1 CE.

9 BAREA, J. «Papeles de Economía española» n. o 69, 1996, pág. 56.

${ }_{10}$ WOLF, C. Mercados o Gobiernos, IEF, 1995, pág. 70. Citado por Barea, J. Op. cit.

${ }_{11}$ STIGLITZ, J.F. Análisis económico de la sanidad. Generalitat de Catalunya. 1994. Citado por Barea, J. Op. cit. 
b) Necesidad de alcanzar la eficacia y la eficiencia para hacer sostenible el SNS

Cualquier solución que se busque para reorientar el Sistema Nacional de Salud, debería estar fundamentado sobre las premisas de financiar la producción y no el coste, sea el que sea, de mantener los centros sanitarios.

Para que el coste de producción de las unidades de prestación de los servicios sanitarios pueda considerarse eficiente, se requieren dos condiciones:

1. Ampliar el número de centros sanitarios competentes, públicos y privados, que constituyan una gran fuente de provisión en competencia eficiente, compatible con la eficacia, que permita garantizar la cantidad de producción, su calidad y el tiempo demandado por los asegurados.

2. Para que esto sea posible, hay que dotar a los asegurados de la facultad de libre elección del centro sanitario, público o privado, porque ellos acudirán donde mejor se consideren atendidos en cantidad, calidad y tiempo.

La necesidad de la eficiencia, además de ser una obligación constitucional (31.2.CE), tiene una dimensión ética, porque «la peor de las éticas es la del despilfarro, no siendo eficientes, aun teniendo que reiterar los economistas de la salud hasta la saciedad que el objetivo de la eficacia no es minimizar costes sino mejor servir al bienestar con unos recursos limitados».12

\section{PROPUESTA DE UN MODELO ALTERNATIVO DE FINANCIACIÓN Y PROVISIÓN DE LAS PRESTACIONES SANITARIAS}

\section{III.1. Principios en los que se fundamenta la propuesta}

1. Principios de Eficacia, Eficiencia y Efectividad

Aparentemente, las tres palabras vienen a significar lo mismo. Sin embargo, cuando nos adentramos en el mundo económico y particularmente en el ámbito del empleo de los recursos públicos,

12 LOPEZ-CASASNOVAS, G. Diario Médico, 20-11-2016, pág. 3. 
podemos diferenciarlo mucho más. Así, por ejemplo, es eficaz un antibiótico que consigue acabar con la enfermedad, y sería eficiente, si la consecución de ese objetivo sanitario se alcanza a un mínimo coste, sin alterar la calidad.

La efectividad es la unión de eficacia y eficiencia, es decir, busca lograr un efecto deseado, en el menor tiempo posible y con la menor cantidad de recursos empleados, sin disminuir la calidad. Por ejemplo, una bombilla de bajo consumo es eficiente, pues puede iluminar igual que una de filamento, y por tanto, es igual de eficaz, pero al consumir menos, logra la efectividad. Desde el punto de vista del modelo que proponemos, es necesario que con él se consiga la eficacia, eficiencia y efectividad del Sistema Nacional de Salud. El objetivo de la eficacia, como principio en este modelo, es conseguir la producción de la cantidad de unidades sanitarias demandadas, con la calidad necesaria y en el menor tiempo que cumpla la finalidad pretendida. La cantidad de producción de servicios sanitarios, se concreta en satisfacer la demanda total, según la Cartera de Servicios que incluya el modelo propuesto, a cuyo propósito se aumentan los recursos humanos y de tecnología sanitaria, mediante la concertación de centros sanitarios públicos y privados.

La calidad de la producción de servicios sanitarios, como principio del modelo, requiere, en primer lugar, conseguir la más alta formación en pregrado, postgrado y permanente de los recursos humanos, además de contar con una red de centros concertados, con inmuebles, instalaciones, y equipos tecnológicos sanitarios en fase de vida vigente, a cuyo fin el modelo prevé pagar un precio, tanto por las inversiones no reversibles, como por las tareas de educación y formación, además de un plan financiero para facilitar las nuevas inversiones, en las condiciones más adelante descritas.

El tiempo mínimo de realización de las prestaciones, como principio, pretende hacer desaparecer las interminables listas de espera, con el efecto combinado del aumento de ofertas de centros sanitarios y la facilidad de libre elección de los asegurados, que podrán optar por el centro público o privado concertado, de cualquier punto del territorio español que considere conveniente. Por su parte, la eficiencia del modelo propuesto se basa en financiar, desde la Administración Central del Estado, las prestaciones sanitarias del catálogo, a los precios por unidad de prestación que se determine en el Baremo de Precios, que realizará y actualizará la comisión de expertos que se cree al efecto, aplicable tanto en centros concertados públicos como privados. 
Conseguir la eficiencia no es ahorro de recursos económicos, sino evitar los derroches de los mismos, para aumentar la eficacia del sistema y su correspondiente sostenibilidad. Es una cuestión de ética pública y de cumplimiento de lo dispuesto en el artículo 31.2. de la Constitución española para mejor servir al bienestar con unos recursos limitados: «Artículo 31.2. El gasto público realizará una asignación equitativa de los recursos públicos, y su programación y ejecución responderán a los criterios de eficiencia y economía». «Defender lo público no es defender el control político. Defender lo público es dar mejor servicio, con más calidad y con coste más eficiente, asegurando la sostenibilidad, la inversión y el empleo. Como se hace en toda la OCDE, con modelos de colaboración público-privadas eficaces» ${ }^{13}$.

Con la aparición de lo que Peter Drucker ${ }^{14}$ denominó trabajo del conocimiento, se da el significado más ajustado de las palabras de eficacia, eficiencia y efectividad. Para Peter Drucker en el trabajo manual característico de la era industrial, la clave fue la mejora de la productividad, es decir, la mejora de la eficiencia. Los avances en productividad del último medio siglo xx han permitido alcanzar los niveles actuales de desarrollo económico y social. Ahora, en el trabajo del conocimiento, lo importante es alcanzar la efectividad, aunque como decía este autor, en efectividad estamos «en pañales». «Los gerentes tendrán que convertirse en empresarios, tendrán que aprender a construir y administrar organizaciones innovadoras ${ }^{15}$ para alcanzar la eficacia, la eficiencia y, en definitiva, la efectividad de los recursos públicos destinados a financiar la provisión de las prestaciones sanitarias para hacer efectivo el derecho de protección de la salud.

\section{Principios de Competencia y libre elección del asegurado}

En el modelo de financiación y provisión de las prestaciones sanitarias que proponemos, es una condición necesaria la existencia de un cierto equilibrio ente demandantes de las prestaciones (los «asegurados») y los prestadores de los servicios sanitarios (los centros concertados, públicos y privados). Al tener los «asegurados» la libertad de elección de centro para ser atendidos, y el hecho de que los ingresos de los centros serán los que cada uno de ellos obtengan

${ }^{13}$ LACALLE, D. Diario $A B C, 01-05-17$, pág. 3.

${ }^{14}$ DRUCKER, P. «La Gerencia. Tareas, responsabilidades y prácticas». 4. a ed, p. 23, 1981. Editorial Ateneo. Buenos Aires.

${ }^{15}$ Ibidem, p. 22. 
con la facturación de las prestaciones sanitarias prestadas, se produce una situación de competencia entre centros concertados, que es el fundamento que da sustento a la eficiencia. Sin competencia equilibrada no se puede concebir la eficiencia y la efectividad de los recursos públicos empleados en financiar el derecho de protección de la salud. Cuando planteamos aquí la competencia, no estamos proponiendo una libre competencia, sinónimo de libre mercado, donde la fundamental orientación para tomar las decisiones económicas proviene de los precios. En la propuesta de nuevo modelo de financiación y provisión de las prestaciones sanitarias que estamos haciendo, el factor precio queda excluido en el principio de competencia, al venir predefinido desde la Administración Central el Baremo de Precios de las distintas unidades de prestación de servicios sanitarios. Por ello el principio de competencia se refiere, fundamentalmente, a la necesidad que tendrán los centros concertados en ser «competentes» en el desempeño de la gestión asistencial en calidad, tiempo y cantidad, para atraer a los asegurados, y obtener así unos ingresos anuales que les permita hacer viable la entidad. Este sistema de competencia imperfecta, al excluir la competencia producida por el factor precio, permite la transformación de los centros públicos en centros públicos concertados con ciertas ventajas competitivas por el buen nivel asistencial que, en general, ya tienen. En este modelo es necesario un cambio de mentalidad, especialmente en los equipos directivos de centros públicos, para pasar de una «gestión presupuestaria» a una "gestión de resultados», lo que implica un cambio de cultura de gestión de todos los recursos (humanos, tecnológicos, retributivos, de inversiones, compras, proveedores, etc.). «España puede mejorar, sin duda. Por ejemplo, haciendo que los hospitales compitan entre sí, de manera que los pacientes puedan elegir siempre el que le brinde mejor calidad.» ${ }^{16}$

$\mathrm{Al}$ otorgarle a los demandantes (los asegurados) la facultad de libre elección de centro sanitario donde será atendido, la decisión que adopte al acudir a uno en lugar de a otro, no estará influida por el factor precio, que estará previamente fijado y lo soportará la administración central, sino que la tomará en función de donde encuentre las mejores expectativas de recibir una asistencia personal y sanitaria de calidad.

La libre elección de centro por los asegurados se debe concretar cuando se implante el modelo propuesto, a título meramente enunciativo, sugerimos algunos criterios: Libre elección de Atención Pri-

${ }^{16}$ SCHEFFLER, R. Profesor de Berkeley, California (EE.UU.). Diario $A B C S a$ lud. 16-09-17, pág. 8. 
maria. (AP), Libre elección de Atención Urgencias, Libre elección de Atención Especializada (AE), Libre elección de Atención Pruebas Diagnósticas, previa prescripción, según prueba, de AP y AE, Libre elección de Atenciones Terapéuticas, previa prescripción de AE, Libre elección de Centro Quirúrgico y Hospitalización, previa prescripción de AE.

\section{III.2. Diagrama y características básicas del modelo}

El Estado, en virtud de lo establecido en el artículo 149.1.16 de la Constitución Española, goza de las facultades de establecer los principios y criterios sustantivos que permitan conferir al sistema sanitario las características generales y comunes que sean fundamento de los servicios sanitarios en todo el territorio del Estado. Será necesario modificar en algunos artículos, tanto la Ley 14/1986, General de Sanidad y la legislación concordante y complementaria que la desarrolla, así como ciertas disposiciones autonómicas que pudieran ser afectadas.

Como puede verse en el diagrama, el modelo propuesto se caracteriza por separar la financiación del derecho de la protección a la salud, de la provisión de las prestaciones sanitarias de tal derecho. Desde el punto de vista de la financiación es necesario revertir esta competencia desde las Comunidades Autónomas, avocándola a la Administración General del Estado para incluirla como partida de los Presupuestos del Estado, que sería gestionada dentro del Sistema Nacional de Salud que se propone. Desde el Sistema Nacional de Salud se financiaría la provisión de las prestaciones sanitarias, mediante un sistema de financiación de la producción de las prestaciones sanitarias realizadas en una red de centros públicos y privados, previamente concertados. La separación entre financiador, comprador y productor de los servicios sanitarios ha sido propuesta por varios autores, entre otros Barea Tejeiro y Gómez Ciria. ${ }^{17}$

17 BAREA TEJEIRO, J. y GÓMEZ CIRIA, A. «El problema de la eficacia del sector público en España. Especial consideración de la Sanidad.» Instituto de Estudios Económicos. Madrid. 1994. 


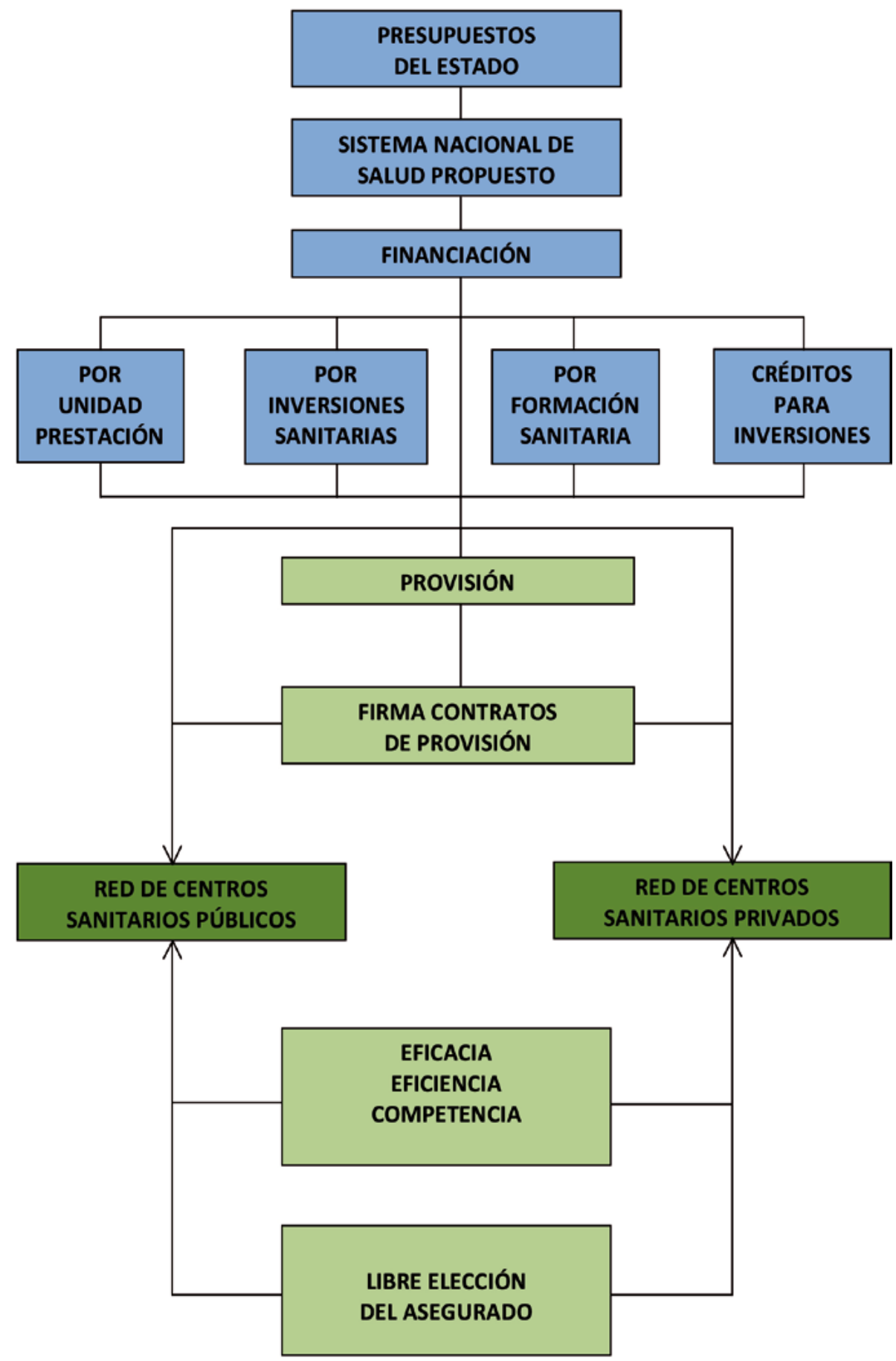

Fuente: elaboración propia. José Jerez Iglesias.

(c) UNED. Revista de Derecho UNED, núm. 23, 2018 
El precio a pagar por la provisión de las prestaciones sanitarias será fijado dentro de un Baremo de Precios a financiar, que será revisado y actualizado para cada anualidad con los criterios técnicos, profesionales y económicos que correspondan. El precio a financiar comprenderá, en su caso, los siguientes conceptos desglosados: Precio por prestación sanitaria básica (PSB), Precio por inversiones no reversibles (PI), Precio por educación y formación (PFS).

Cada centro concertado, público o privado, realizará un presupuesto anual de actividad con la estimación de sus Ingresos, Gastos y Resultados, debiendo tener autonomía absoluta de gestión, respondiendo de la misma ante sus titulares, que en el caso de los centros públicos serán por lo general las Comunidades Autónomas. El resultado anual de cada centro público (positivo o negativo) corresponde al titular del mismo, quien deberá determinar en su normativa propia el destino del mismo. Simultáneamente formalizarán sus planes anuales de inversiones de inmuebles, instalaciones, equipos, mobiliario, etc, con un horizonte temporal de cinco años.

Cada centro, público o privado, deberá ser concertado, mediante la firma de un Contrato de Concierto de Provisión, que incluirá los Planes de Inversiones y de Educación y Formación. Los Planes de Inversiones, una vez aprobados por el Sistema Nacional de Salud, serán financiados parcialmente por un Crédito de Tesorería, en las condiciones financieras y de garantías necesarias, así como en consideración al nivel de endeudamiento sobre PIB del titular público superior del centro. La amortización de estos créditos se realizará reteniendo y abonando en la cuenta del crédito, la cuota por inversiones que se asocie a la prestación sanitaria. En el caso de los centros privados, no se contempla el sistema de créditos por inversiones, y por ello no se le retendrá la cuota de inversiones. El montante de Crédito de Tesorería deberá ser asignado en función de criterios objetivos de actividad prevista con las inversiones según cada centro, y en función del Plan de Inversiones anual aprobado. Con este sistema de financiación se pretende combatir la obsolescencia tecnológica de las instalaciones y equipos de los centros para ganar en eficiencia.

Los Planes de Educación y Formación, se actualizarán anualmente y deben incluir las prácticas de pregrado de la rama sanitaria cuyos planes de estudio así lo determinan, y la formación de los distintos tipos de especialidades que deban superar una estancia como residente en un centro sanitario, Mir (Médico interno residente), Eir (Enfermero interno residente), Pir (Psicólogo), Fir (Farmacia), Bir (Biología), etc. La dotación económica debe tener la condición de finalista a cuyo fin cada Centro Concertado, solo podrá disponer de 
estos fondos para el cumplimiento de la actividad educativa y de formación, por ejemplo, para la retribución de Tutores, dotación de aulas, seminarios, conferencias, congresos, material de prácticas, audiovisuales, etc. El precio por la actividad educativa y de formación, alternativamente, puede asociarse al precio por la prestación sanitaria básica (PSB), o abonarse independientemente de las prestaciones que realice cada centro concertado, y estimarlo por un sistema capitativo, de acuerdo con el número de personas que reciben la formación y el nivel de los mismos, por ejemplo, prácticas de pregrado, formación de residentes (R-1, R-2, R-3, R-4, etc.) multiplicado por un valor capitativo para cada uno de los grupos.

\section{III.3. Centros concertados públicos y privados}

Los centros concertados públicos podrán optar a la concertación de las unidades de prestación sanitaria que cada uno de esos centros pueda acreditar. De tal forma que en una Comunidad Autónoma concreta, en la que en la actualidad existe una pluralidad de centros sanitarios públicos, con atenciones prestacionales diversas, deberán, en primer lugar, definir y concretar cuantas unidades empresariales públicas van a constituir y con qué cartera de servicios. Este primer paso permitirá, a cada autonomía, poder dotar de personalidad jurídica y patrimonial independiente y, consecuentemente de su gestión sanitaria, económica y financiera, que permita su mejor control y seguimiento por la Comunidad Autónoma.

A cada una de estas unidades empresariales se les adscribirá parte de los centros asistenciales existentes, siguiendo los criterios de volumen, complementariedad, zonificación, etc. Por ejemplo, una unidad empresarial podría constituirse con un Hospital General, con todos sus servicios, centros de salud asociados y complementarios dentro de una zona o área de salud, sin adscripción alguna de asegurados, ya que estos tendrán libertad para elegir el centro donde deseen ser atendidos, y a su vez los centros podrán atender a cualquier asegurado de otros territorios de la Comunidad o fuera de ella, facturando las unidades de prestación sanitarias realizadas. Por tanto, los sujetos jurídicos susceptibles de ser concertados, serán las unidades empresariales que cada autonomía decida constituir, y ésta unidad empresarial será la que formalizará el Contrato de Concierto y realizará sus Planes de Inversiones y de Educación y Formación, solicitará los créditos para inversiones de acuerdo con sus respectivos planes de inversión, etc. Naturalmente, cada Comunidad Autónoma, como titular del capital social de cada unidad empresarial, designará 
la composición del Órgano de Administración, supervisará el nombramiento de los correspondientes gestores, controlará la gestión de los mismos, aprobará sus planes de inversiones y de formación, así como sus Cuentas Anuales, y orientará las políticas que mejoren la calidad asistencial.

Los centros concertados privados podrán optar a la concertación de las unidades de prestación sanitaria que cada uno de esos centros pueda acreditar. Cada centro privado puede optar por concertar una o varias unidades empresariales, aunque constituyan una única unidad desde el punto de vista mercantil, bajo la misma denominación social, si a sus intereses de gestión sanitaria, económica y financiera le fuese conveniente. Ello implicaría la posibilidad de concertar algunos centros de actividad y otros no. Cada una de estas unidades empresariales tendría adscritos una parte de los centros asistenciales existentes para ser concertados, si bien jurídica y patrimonialmente se concierta con la denominación social matriz. Tanto los centros públicos como los privados a partir de la unidad empresarial, de cada una de ellas, concretará su cartera de servicios sanitarios, los cuales sugerimos distinguir así:

1. ACTO MÉDICO UNITARIO AMBULATORIO: 1.1. Consulta de atención primaria, 1.2. Consulta de atención especializada., 1.3. Consulta de atención de urgencias. 2. ACTO MÉDICO POR PROCESO AMBULATORIO: 2.1. Prueba diagnóstica, 2.2. Atención terapéutica, 2.3. Cirugía ambulatoria. 3. ACTO MÉDICO POR PROCESO HOSPITALARIO: 3.1. Cirugía por proceso hospitalario, 3.2. Atención médica por proceso hospitalario.

Existe la posibilidad de concertar capitativos para atención de urgencias en ciertas áreas rurales o urbanas. El acto médico retribuido por capitativo es una herramienta que se puede utilizar para obtener una doble garantía, por un lado al asegurado se le garantiza un servicio de urgencias 24 horas en zonas de baja población, donde objetivamente podría no existir ese servicio, y al mismo tiempo al centro concertado se le garantiza unos ingresos fijos, una cantidad por asegurado del área, que recibirá sea cual sea el nivel de actividad asistencial prestada.

\section{III.4. La financiación}

Una característica del modelo propuesto es que se asignará en el Baremo de Precios, para cada una de las prestaciones sanitarias concertadas, una parte agregable al precio de dicha prestación (PSB), que se denominará precio por inversiones no reversibles (PI). La 
cuantía de este precio se determinará por una comisión de expertos y formará parte del Baremo de Precios.

Una de las cuatro patas básicas del modelo propuesto, es la financiación de las inversiones para la adquisición de instalaciones de tecnología sanitarias e inmuebles, a cuyo fin se propone la creación de un Banco de Crédito Sanitario para financiar exclusivamente las inversiones de centros concertados públicos.

Los centros concertados públicos que hayan firmado un Contrato de Concierto con la Administración Central estarían en condiciones de presentar una solicitud de crédito para financiar inversiones, mediante la suscripción de un Plan de Inversiones Sanitarias, justificando la necesidad de las mismas y el plan de amortización. La forma ordinaria de reembolso de los créditos sanitarios se realizará mediante retención de la parte agregable al precio básico de la prestación sanitaria destinado a la financiación de inversiones (PI), de forma que esa parte del precio de la prestación sanitaria será devengada con cada prestación y retenida su cuantía para ser abonada en la cuenta de crédito con destino a su amortización financiera.

\section{III.5. Una propuesta de copago}

La situación actual de copago en España se refiere al copago de medicamentos por receta en régimen ambulatorio, cuya última regulación se ha efectuado por el Real Decreto 16/2012, de 20 de abril, de medidas urgentes para garantizar la sostenibilidad del Sistema Nacional de Salud. En el cuadro siguiente se refleja la situación actual y la anterior a la entrada en vigor del RD 16/2012:

A la vista del cuadro-resumen podemos establecer las siguientes conclusiones:

1. En el grupo de pensionistas se observa una falta de equidad de aportación por la ausencia de progresividad del sistema, con un tramo demasiado amplio entre 18.000 euros, y 100.000 euros, y especialmente con las rentas medias/bajas, teniendo en cuenta que este grupo no aportaba nada antes del 01/07/2012, 2. En el grupo de personas sin recursos (con rentas de integración, pensiones no contributivas, síndromes tóxicos o personas con discapacidad), la situación ha mejorado al no tener que aportar nada, frente al $40 \%$ que aportaban antes del 01/07/12 estas personas, 3. Los parados sin subsidio mejoran también al no tener que aportar nada, frente al $40 \%$ que aportaban estas personas antes del 01/07/12, 4. Las personas con tratamien- 
tos por accidentes de trabajo o enfermedad profesional, permanecen igual, al no tener que pagar nada, 5. Personas con tratamientos crónicos para los medicamentos pertenecientes a los grupos ATC y productos sanitarios de aportación reducida, seguirán pagando el 10\%, pasando el límite mensual de pago por receta de 2,64 euros a 4,26 euros, 6. Personas asegurados en general, se mantiene en un $40 \%$ para rentas inferiores a 18.000 €/año, sube del 40 al 50\% para rentas entre $18.000 € /$ año y $100.000 € /$ año, y sube del $40 \%$ al $60 \%$ para el grupo de rentas superior a $100.000 €$ / año. Aquí se observa también falta de equidad en los tramos desde rentas inferiores a $18.000 € /$ año hasta los $100.000 € /$ año, al carecer de una adecuada progresividad, 7. Grupo de mutualistas y clases pasivas de MUFACE, ISFAS Y MUGEJU, se mantiene en una aportación del 30\%.

Cuadro 1. Copago de medicamentos en el SNS.

Datos a enero de 2015

\begin{tabular}{|c|c|c|c|}
\hline \multirow{2}{*}{ Grupo } & \multirow{2}{*}{$\begin{array}{c}\text { Antes } \\
\text { RD 16/2012 }\end{array}$} & \multicolumn{2}{|c|}{ Desde 01-07-12 } \\
\hline & & General & Límite(1) \\
\hline \multicolumn{4}{|l|}{ 1. PENSIONISTAS } \\
\hline a) Menos $18.000 € /$ año & $0 \%$ & $10 \%$ & $8,23 €$ \\
\hline b) Entre $18.000-100.000 € /$ año & $0 \%$ & $10 \%$ & $18,52 €$ \\
\hline c) Más 100.000 €/año & $0 \%$ & $60 \%$ & $61,75 €$ \\
\hline \multicolumn{4}{|l|}{ 2. PERSONAS SIN RECURSOS } \\
\hline a) Con Renta integración & $40 \%$ & $0 \%$ & $0,00 €$ \\
\hline b) Con Pensiones no contributivas & $0 \%$ & $0 \%$ & $0,00 €$ \\
\hline c) Con síndrome tóxico, y discapacitado & $0 \%$ & $0 \%$ & $0,00 €$ \\
\hline \multicolumn{4}{|l|}{ 3. PARADOS } \\
\hline a) Sin subsidios & $40 \%$ & $0 \%$ & $0,00 €$ \\
\hline 4. ACCID. TRAB./ENFERM. PROF. & $0 \%$ & $0 \%$ & $0,00 €$ \\
\hline 5. CRÓNICOS & $\begin{array}{c}10 \% \\
\operatorname{lm} 2,64 €\end{array}$ & $10 \%$ & $4,26 €$ \\
\hline \multicolumn{4}{|l|}{ 6. GENERAL } \\
\hline a) Menos $18.000 € /$ año & $40 \%$ & $40 \%$ & Sin límite \\
\hline b) Entre $18.000-100.000 € /$ año & $40 \%$ & $50 \%$ & Sin límite \\
\hline c) Más 100.000 €/año & $40 \%$ & $60 \%$ & Sin límite \\
\hline 7. MUTUALISTAS & $30 \%$ & $30 \%$ & Sin límite \\
\hline
\end{tabular}

(1) Límite máximo de aportación por tratamiento de larga duración (euros/mes).

Fuente: Elaboración propia. 
Valoración situación actual. El sistema actual no es equitativo y habría que reconsiderar dos aspectos fundamentales: UNO. Considerar a todos los españoles en un plano de igualdad, con independencia de ser o no funcionarios y de estar en activo o jubilado, en función de la renta anual percibida. DOS. Los tramos actuales para aportaciones en copago farmacéutico en función de las rentas percibidas de menos de 18.000 €/año, entre $18.000 € /$ año y 100.000 €/año, y más de $100.000 € / a n ̃ o$, son demasiado amplios, sobre todo el tramo de 18.000-100.000 €, por lo que tienen falta de equidad. TRES. Hay que reconocer el importante efecto que ha tenido la modificación de las aportaciones en copago farmacéutico, que ha representado un ahorro en la factura de este gasto, desde la aplicación del RD 16/2012, de las medidas adoptadas para garantizar la sostenibilidad del SNS hasta el 31 de diciembre de 2015, que se eleva a 5.657,8 millones de euros. El gasto medio por habitante y año ha pasado de 270,3 euros en 2010 a 214,3 euros en $2015 .^{18}$

Alcance de la propuesta. La propuesta que realizamos afecta a tres aspectos de los copagos:

1. Igualdad de todos los ciudadanos respecto de los copagos. En nuestra opinión, resulta más equitativo que los ciudadanos se agrupen en función de sus rentas anuales, es decir, en función de su capacidad de pago, con independencia de su situación laboral de ser funcionario o no, o de estar en edad de ser activo o de jubilado, o de la procedencia de sus rentas. El sistema actual es discriminatorio en favor de funcionarios y jubilados, sin que exista ninguna razón objetiva de peso que lo justifique.

2. Ampliar los tramos de rentas anuales, agrupando a todos los ciudadanos en los siguientes tramos:

${ }_{18}$ Ministerio de Sanidad, Servicios Sociales e Igualdad. Informe anual del Sistema Nacional de Salud, 2016, pág. 238. 
Cuadro 2. Propuesta nuevos tramos copago, según renta

\begin{tabular}{|c|c|}
\hline Grupos & Integrantes \\
\hline A & $\begin{array}{l}\text { - Rentas de Integración. Pensiones no contributivas. Síndrome tóxico. } \\
\text { Discapacidad. Sin subsidios. }\end{array}$ \\
\hline $\mathrm{B}$ & —Crónicos y Rentas hasta 18.000 €/año. \\
\hline $\mathrm{C}$ & —Rentas desde 18.001 €/año hasta 36.000 €/año. \\
\hline $\mathrm{D}$ & —Rentas desde 36.001 €/año hasta 54.000 €/año. \\
\hline $\mathrm{E}$ & —Rentas desde 54.001 €/año hasta 72.000 €/año. \\
\hline $\mathrm{F}$ & —Rentas de 72.001 €/año hasta 90.000 €/año. \\
\hline G & —Más de 90.001 €/año. \\
\hline
\end{tabular}

A partir de los nuevos tramos de rentas nuestra propuesta de copago farmacéutico queda según se refleja en el siguiente cuadro:

Cuadro 3. Propuesta nuevos porcentajes de copagos farmacéuticos

\begin{tabular}{c|cc}
\hline Grupos & \multicolumn{1}{|c}{ Integrantes } & Copago \\
\hline A & - Rentas de Integración. Pensiones no contributivas. Síndrome & $20 \%$ \\
& tóxico. Discapacidad. Sin subsidios. & \\
B & - Crónicos y Rentas hasta 18.000 €/año. & $30 \%$ \\
C & - Rentas desde 18.001 €/año hasta 36.000 €/año. & $40 \%$ \\
D & - Rentas desde 36.001 €/año hasta 54.000 €/año. & $50 \%$ \\
E & -Rentas desde 54.001 €/año hasta 72.000 €/año. & $60 \%$ \\
F & -Rentas de 72.001 €/año hasta 90.000 €/año. & $70 \%$ \\
G & -Más de 90.001 €/año. & $80 \%$ \\
\hline
\end{tabular}

Fuente: elaboración propia.

3. Ampliar los conceptos de copagos. Hasta ahora el copago se refiere al de productos farmacéuticos prescritos por receta ambulatoria. Sin embargo, consideramos que al introducir un sistema donde el ciudadano va a tener libertad de elección de centro donde ser atendido, dentro de los concertados públicos y privados, como contrapeso del posible uso abusivo que pudiera favorecer el modelo, sugerimos ampliar el copago a las siguientes prestaciones sanitarias: Consultas de Atención Primaria (AP), Consultas de Atención Especializada (AE), Consultas de Urgencias (AU), Consultas de Pruebas Diagnósticas (PD), Consultas de Atención Terapéutica (AT). 
Propuesta de copago asistencial. Para tratar de moderar o reducir el abuso o sobreuso del sistema, sugerimos la implementación de un tícket moderador de copago, a partir de un número de atenciones al año exentas en las consultas de Atención Primaria (AP), Atención Especializada (AE), Urgencias (AU), Pruebas Diagnósticas (PD) y Atención Terapéutica (AT). «Será posiblemente un hecho que los copagos vuelvan a aparecer en determinados casos de tratamientos no esenciales. Ello esboza un sistema en el que el usuario va a observar algunos cambios en la forma de atención, pero que si se gestionan adecuadamente pueden resultar positivos. ${ }^{19}$

La idea consiste en asignar un crédito gratuito de tícket para acudir a estas consultas. La asignación del número de tícket será variable en atención a las circunstancias de cada asegurado, especialmente la cronicidad o situaciones puntuales, y que deban ser consideradas como «normales» en cada circunstancia (criterios a fijar por los profesionales competentes), pudiendo ser revisado al alza desde el sistema informático central, según los casos. Cada vez que un asegurado asiste a una consulta consume un número de tickets variable, según consulta y grupo de renta donde se encuentre clasificado. Una vez agotado su crédito de tickets, debe adquirir los que necesite. El procedimiento parte del principio de la existencia de un sistema informático constituido por toda la red de centros concertados que incluya a todos los asegurados con derecho a las prestaciones sanitarias. Es el caso del actual Sistema Nacional de Salud que cuenta con el procedimiento e-salud de identificación electrónica de los usuarios que integran el Sistema de Historia Clínica Digital y el Sistema de Receta Electrónica, como instrumentos vertebradores de los agentes del SNS, con un fuerte componente de Tecnología de la Información (TIC), lo que permitirá adoptar sin grandes cambios el procedimiento para los tickets de consultas. ${ }^{20} \mathrm{~A}$ cada titular con derecho a las prestaciones sanitarias se le tiene que proveer de una tarjeta sanitaria individual, con validez para todo el territorio nacional, con la que acudirá para ser atendido en los respectivos centros sanitarios. La tarjeta debe incorporar, o dar acceso, a la información actualizada sobre las siguientes cuestiones: Datos administrativos personales del asegurado y su clasificación para copago en función de su renta, Cuenta de tickets para consultas, con acceso del asegurado y de los centros asistenciales, e Historia clínica personal, con acceso restringido a personas identificadas y con clave. A efecto de control

19 MARCOS DEL CANO, A.M. y MÉNDEZ MARTÍN, J. Op. cit.

${ }^{20}$ Ministerio de Sanidad, Servicios Sociales e Igualdad. Informe anual del Sistema Nacional de Salud, 2016, pag. 269. 
de los tickets, cada vez que el asegurado acuda a una consulta deberá entregar su tarjeta, firmar el justificante que corresponda y se le restara automáticamente el número de tickets consumido, en función del tipo de consulta y de su grupo de renta. Una vez que al asegurado se le agote sus tickets gratuitos, tiene que recargar su tarjeta con tickets de 0,50 € cada uno, en la cantidad que considere necesario. El recargo de la tarjeta se podrá efectuar en máquinas instaladas en los principales centros sanitarios, bien mediante dinero en efectivo o con tarjeta de débito o crédito. Un modelo conocido de este tipo de máquinas de recargo de tarjetas, es el instalado en la red de estaciones del Metro de Madrid.

Como resumen de la propuesta de copagos de farmacia y asistencia hemos elaborado un cuadro de las sugerencias que se muestra a continuación:

Cuadro 4. Resumen sugerencias de copagos farmacéuticos y asistenciales

\begin{tabular}{crrrrrr}
\hline \multicolumn{7}{c}{ Copagos $^{(1)}$} \\
\hline Grupos & Farma $^{(2)}$ & \multicolumn{1}{c}{ A.P. } & \multicolumn{1}{c}{ A.E. } & \multicolumn{1}{c}{ A.U. } & P.D. $^{(3)}$ & A.T. $^{(3)}$ \\
\hline A & $20 \%$ & 1 tícket & 2 tíckets & 4 tíckets & 4 tíckets & 4 tíckets \\
B & $30 \%$ & 2 tíckets & 3 tíckets & 5 tíckets & 5 tíckets & 5 tíckets \\
C & $40 \%$ & 3 tíckets & 4 tíckets & 6 tíckets & 6 tíckets & 6 tíckets \\
D & $50 \%$ & 4 tíckets & 5 tíckets & 7 tíckets & 7 tíckets & 7 tíckets \\
E & $60 \%$ & 6 tíckets & 7 tíckets & 9 tíckets & 9 tíckets & 9 tíckets \\
F & $70 \%$ & 8 tíckets & 9 tíckets & 11 tíckets & 11 tíckets & 11 tíckets \\
G & $80 \%$ & 10 tíckets & 11 tíckets & 13 tíckets & 13 tíckets & 13 tíckets \\
\hline
\end{tabular}

(1) A partir de haber consumido un crédito previamente establecido para cada asegurado.

(2) Para receta en régimen ambulatorio.

(3) Máximo por prueba, puede ser inferior según tipo de prueba o asistencia terapéutica, a modular técnicamente.

Fuente: elaboración propia.

\section{III.6. Ventajas del modelo propuesto}

Hemos analizado las ventajas, es decir, los aspectos positivos o beneficiosos que se conseguirían con la implantación del modelo de financiación y provisión propuesto, respecto de la problemática 
existente de ineficiencias del actual modelo y hemos identificado las más significativas, las cuales seleccionamos de forma resumida:

1. Garantiza la igualdad de los españoles. Con la implantación de la libertad de elección del asegurado para acudir al centro concertado, público o privado, que desee dentro del territorio español se lleva a puro efecto lo dispuesto en el artículo 14 de la Constitución española que dice: "Artículo 14. Los españoles son iguales ante la ley, sin que pueda prevalecer discriminación alguna por razón de nacimiento, raza, sexo, religión, opinión o cualquier otra condición o circunstancia personal o social». Esta igualdad de todos los españoles implica poder disfrutar del mismo contenido de la cartera de servicios sanitarios y de recibir la debida atención sanitaria en igualdad de trato en cualquier punto del territorio español, desapareciendo las actuales dificultades de compensación entre los diecisiete sistemas autonómicos.

2. Asigna equitativamente los recursos públicos. El modelo de provisión de las prestaciones sanitarias que proponemos se caracteriza por separar la financiación de la provisión, pasándose a financiar la provisión de las unidades de prestaciones sanitarias realizadas en una red de centros públicos y privados, previamente concertados. Con este procedimiento se abandona financiar el coste de los centros públicos, fuese éste el que fuese, y se pasa a financiar el precio de la producción en un entorno competitivo, lo que supone cumplir con lo dispuesto en el artículo 31.2. de la Constitución española para mejor servir al bienestar con unos recursos limitados: «Artículo 31.2. El gasto público realizará una asignación equitativa de los recursos públicos, y su programación y ejecución responderán a los criterios de eficiencia y economía.»

3. Garantiza la sostenibilidad del SNS. Al introducir en el SNS la gestión de la financiación de la provisión de forma centralizada, se financia el mismo coste por unidad de prestación sanitaria tanto en centros concertados públicos como privados. Los centros concertados, tendrán que competir entre ellos, no en precio, pero sí en ser competentes en alcanzar la mejor calidad asistencial, aplicando los principios de eficacia (productividad) y eficiencia (costes justos), lo que beneficiará al SNS al hacer desaparecer todos los costes asociados a las ineficiencias, garantizando así su evolución y sostenibilidad.

4. Mejora de la financiación y gestión autonómica. El modelo actual de financiación de las Comunidades Autónomas es motivo de insatisfacción general. Uno de los capítulos que absorbe ma- 
yor cantidad de recursos es el correspondiente a la financiación de las prestaciones sanitarias del derecho a la protección de la salud de los ciudadanos.

En términos de PIB, Extremadura tiene un porcentaje del 9,4\%, seguida de Murcia con el 7,7\% y de Asturias con el 7,3\%, que son las comunidades con mayor porcentaje. Por el contrario, Madrid con el $3,8 \%$, Cataluña con el 4,7 y Baleares con el 5,1\% son las de menor porcentaje de PIB. En valores absolutos, el 44,4\% del gasto sanitario público del sector es originado por tres Comunidades Autónomas: Cataluña, Andalucía y Madrid, con 9.205, 8.737 y 7.423 millones de euros, respectivamente. La Rioja, Cantabria y Navarra son las Comunidades que generaron el gasto más bajo. En relación a su población, las Comunidades con más gasto sanitario público son País Vasco con $1.582 € /$ habitantes, Extremadura con $1.448 € /$ habitantes y Navarra con $1.435 € /$ habitantes. ${ }^{21}$ Estas ingentes aportaciones presupuestarias, además, resultan insuficientes y están generando retrasos en los cambios necesarios de inmuebles, instalaciones, equipos de tecnología sanitaria, abocando al sistema a una obsolescencia galopante de difícil abordaje. Con el sistema actual, todas las autonomías encuentran dificultades para financiar las demás competencias que tienen atribuidas, con el consiguiente perjuicio para la ciudadanía y el desarrollo social y económico de su población y territorio.

Con el modelo de financiación propuesto se resolvería una parte muy importante de la financiación autonómica, ayudando a las Comunidades Autónomas a centrarse en la gestión de los centros públicos concertados, dotándolas de instrumentos modernos para abordar las políticas de personal, equipamientos, etc., en busca de su propia eficacia y eficiencia con resultados medibles y comparables, que harán progresar las respectivas autonomías.

\section{Facilita la integración de MUFACE, MUGEJU e ISFAS} en el SNS. Como es conocido, actualmente alrededor de dos millones de personas están integrados en la Mutua de Funcionarios Civiles del Estado (MUFACE), la Mutua General de la Judicatura (MUGEJU) y los miembros del Ejército y Fuerzas Armadas (ISFAS). En lo que se refiere a la asistencia sanitaria, incluyendo la farmacéutica, Muface, Mugeju e Isfas forman parte del Sistema Nacional de Salud, en su calidad de entidades gestoras del Régimen Especial

${ }^{21}$ Fuente: Ministerio de Sanidad, Servicios Sociales e Igualdad. Estadística de Gasto Sanitario Público. Pág. 269. 
de la Seguridad Social de los funcionarios. La asistencia sanitaria se proporciona, a elección del mutualista, bien en el sistema sanitario público, o bien a través de los medios de las entidades de seguro libre que hayan concertado con las Mutualidades dichas asistencias, por ejemplo, Adeslas, Asisa, DKV, Caser, etc. Hasta ahora, el sistema ha funcionado aceptablemente bien, pero no está exento de problemas, especialmente por lo que supone de cierta desigualdad de unos españoles (la mayoría) a otros (los funcionarios) dado que estos últimos tienen el privilegio de poder elegir el sistema de prestación sanitaria que quieran. Este sistema de prestación sanitaria es el elegido por el ochenta por ciento de los mutualistas, aún a costa de retenerles una pequeña cantidad en sus nóminas, mientras que el veinte por ciento opta por la atención sanitaria pública. ${ }^{22}$

Con la propuesta que realizamos de nuevo modelo de financiación y provisión de las prestaciones sanitarias, con libre elección de los asegurados, tanto en centros concertados públicos como en centros concertados privados, carecería de sentido alguno seguir manteniendo el Régimen Especial de la Seguridad Social de los funcionarios, al poderse integrar de pleno en el SNS y poder disfrutar al mismo tiempo de la sanidad pública y de la privada.

6. Desaparecen las listas de espera. Con la concertación de centros públicos y privados, en un entorno de libre elección de los asegurados, aumentará la oferta de centros sanitarios en cuantía muy significativa. Una estimación prudente podría ser que, junto a la concertación del cien por cien de los centros públicos, se pudiera concertar una cantidad de centros privados equivalente a un cincuenta por ciento de la capacidad asistencial de los centros públicos concertados, especialmente en atención primaria y especializada, pruebas diagnósticas, asistencia terapéutica, cirugía ambulatoria y con hospitalización, etc.

La ventaja de la desaparición de las listas de espera supondrá, además de la mejora de la calidad asistencial y consecuentemente del bienestar de las personas, un ahorro en número de consultas, repetición de pruebas, atenciones de urgencias, consumo de medicamentos, menos horas de trabajos perdidas por bajas asistenciales... etc.

7. Facilita abordar mejor la obsolescencia tecnológica sanitaria. España no cumple con las directrices europeas del COCIR (Comité Europeo de Coordinación de la Industria de Electro-me-

${ }^{22}$ Informe de Situación sobre MUFACE. CSIF. Madrid. 20-01-17, pág. 12. 
dicina, Radiología y Cuidado de Salud). Estamos en los últimos lugares en Europa en obsolescencia, por ejemplo, quinto lugar por la cola en TAC y en último lugar en Resonancia Magnética. "El parque tecnológico de las comunidades es el más antiguo de las últimas décadas. Habría que invertir un total de 1.400 millones de euros en cuatro años para cumplir las directrices de COCIR. ${ }^{23}$

8. Aumenta la responsabilidad y satisfacción de los asegurados. La libre elección del asegurado le otorga un poder de responsabilidad en su decisión, al tener varias opciones a su disposición, lo que implicará un mayor nivel de satisfacción por tener cubiertas sus expectativas de forma razonable. Esta libre elección del asegurado facilitará también poder establecer, como contrapeso, un sistema de copagos mínimos, establecido con criterios racionales de equidad, mínimos exentos para ciertas rentas, etc con el objeto de frenar o reducir la demanda abusiva en muchas prestaciones, por ejemplo, los servicios de urgencias.

9. Aumenta la calidad asistencial. Con el sistema de centros concertados públicos y privados, con libre elección del asegurado en todo el territorio español, se posibilita que todos los centros asistenciales mejoren las condiciones de actitud y aptitud de todo su personal, para alcanzar las cotas más altas de satisfacción de los usuarios y del personal. Es razonable pensar que los centros implementarán sistemas de retribución variable asociados al nivel de satisfacción de los usuarios y a la eficacia y eficiencia alcanzada en su trabajo, y en sus resultados, lo que necesariamente se traduce en un aumento de la calidad asistencial general.

10. Facilita la introducción del copago. La implantación del copago para prestaciones sanitarias de consultas de Atención Primaria (AP), de Atención Especializada (AE), de Urgencias (AU), de Pruebas Diagnósticas (AD) y de Atención Terapéutica (AT), deviene imprescindible como contrapeso a la libre elección del asegurado. Además, el copago resulta también una herramienta para evitar el abuso asistencial, inducido o no, que se presenta como un riesgo del modelo propuesto.

${ }^{23}$ LÓPEZ CARRASCO, M.L. Presidenta de FENIN. Diario Médico, 04-10 de diciembre de 2017, pág. 5 . 


\section{CONCLUSIONES}

En nuestra opinión el Sistema Nacional de Salud actual, soporta importantes factores que inciden en su falta de sostenibilidad, genera ineficiencias significativas, como listas de espera excesivas, desigual atención a los asegurados fuera de su autonomía de residencia, obsolescencia tecnológica, distorsión de la financiación autonómica entre las más significativas, y, en conclusión, creemos que existen razones suficientes para proponer su reforma.

Hemos objetivado que la propuesta de nuevo modelo que realizamos, es compatible con las disposiciones constitucionales de los artículos 149.1.16 CE, respecto de las facultades del Poder Ejecutivo para llevarla a cabo, $14 \mathrm{CE}$, que garantiza la igualdad de todos los españoles y $31.2 \mathrm{CE}$, que exige asignar equitativamente los recursos públicos con criterios de eficiencia y economía.

Con nuestra propuesta se posibilita una gestión basada en los principios de eficacia y eficiencia, dotando al asegurado de libertad de elección entre centros concertados públicos y privados, que aumenta la responsabilidad de los asegurados por su libertad de elección y, al mismo tiempo, facilita la implementación de un sistema de copago, en contrapeso a la libertad de elección, con una finalidad disuasoria y no recaudatoria.

Del análisis que hemos realizado de las ventajas que se derivarían con la implementación del modelo propuesto, entre otras, como garantizar la igualdad de los españoles, asignación equitativa de los recursos públicos, mejorar la financiación autonómica, hacer desaparecer las listas de espera y la obsolescencia tecnológica, y aumentar la calidad asistencial, podemos concluir que estas ventajas harían desaparecer las ineficiencias que genera el actual SNS y supondría un avance en la modernización de la gestión sanitaria, ganando en eficacia, eficiencia, sostenibilidad y satisfacción de los usuarios.

\section{BIBLIOGRAFÍA}

\section{Autores}

BAREA, José, «Papeles de Economía española», n. 69, 1996.

Barea Tejeiro y Gómez Ciria, A. «El problema de la eficacia del sector público en España». Instituto de Estudios Económicos. Madrid. 1994. 
Drucker, P. «La Gerencia. Tareas, responsabilidades y prácticas». 4. ${ }^{\mathrm{a}}$ ed. 1981.Ed. Ateneo, Buenos Aires, págs. 22 y 23.

Gallete, A. Presidente Seram. Diario Médico, 11-06-17.

LaCALLE, D. Diario $A B C$, 01-05-17.

López Carrasco, M.L. Presidenta Fenin. Diario Médico, 04-10 de diciembre de 2017.

López-Casasnovas, G. Diario Médico, 20-11-16.

Manzanares, L. Diario Médico, 11-06-17.

Marcos del Cano, Ana María y Méndez Martín, J. «El Derecho a la Salud. Perspectivas tras la crisis». Revista de Derecho UNED, n. ${ }^{\circ}$ 19, 2016, págs. 166, 179.

SCHEFFER, R. Profesor Berkeley. ABC Salud, 16-09-17.

STigLitz, J.F. Análisis Económico de la Sanidad. Generalitat de Catalunya, Citado por BAREA, J., "Papeles de Economía española», n. ${ }^{\circ} 69,1996$.

Wolf, C. Mercados o Gobiernos, Ief. 1995. Citado por BAREA, J., «Papeles de Economía española», n. ${ }^{\circ}$ 69, 1996.

\section{Legislación}

CONSTITUCIÓN ESPAÑOLA DE 1978.

Ley Orgánica 1/2011, de 28 de enero, Texto Refundido del Estatuto de Autonomía de la Comunidad Autónoma de Extremadura.

LEY 14/1986, de 25 de abril, General de Sanidad.

LeY 41/2002, Básica Reguladora de la Autonomía del Paciente.

LEY 16/2003, de Cohesión y Calidad del Sistema Nacional de Salud.

LEY 40/2015, de 1 de octubre, Régimen Jurídico del Sector Público.

LEY 9/2017, de 8 de noviembre, de Contratos del Sector Público.

REAL DeCRETO-LEY 16/2012. Exposición de motivos I.

\section{Organismos y prensa}

CSI-F. Informe de Situación sobre Muface. Madrid 20-01-17. 
Ministerio de Sanidad, Servicios Sociales e Igualdad. Subdirección General de Información Sanitaria e Innovación. Informe anual del Sistema Nacional de Salud 2016 y Sistema de Información de Listas de Espera (Sisle).

RAE. Diccionario de la Lengua Española. 21. a edición. 1992. Espasa Calpe, Madrid.

\section{Páginas webs consultadas}

www.redaccionmedica.com 
